\title{
Generation of New Frequencies in Toroid Microcavities
}

\author{
Duygu Akbulut, ${ }^{1}$ Abdullah Tulek, ${ }^{2}$ Mehmet Bayindir ${ }^{1,2}$ \\ ${ }^{I}$ UNAM-Institute of Materials Science and Nanotechnology, 06800 Bilkent, Ankara, Turkey \\ ${ }^{2}$ Department of Physics, Bilkent University, 06800 Bilkent, Ankara, Turkey \\ Tel: (90312) 266 4530,Fax: (90312) 266 4365, e-mail: duyguak@bilkent.edu.tr
}

\begin{abstract}
Microtoroid cavities with ultra high Q-factor have been fabricated using a combined process of photolithography and reflow technique for observing non-linear effects such as generation of new frequency components. For this purpose $\mathrm{SiO}_{2}$ material was used to form the toroidal cavity shape, and chalcogenide material of $\mathrm{As}_{2} \mathrm{~S}_{3}$ maintaining very high nonlinear refractive index was thermally evaporated on top with varying thicknesses. Simulation results of the fabricated structure have exhibited new frequency components around the excitation window of $1520 \mathrm{~nm}$ produced by non-linear interactions.
\end{abstract}

Keywords: Supercontinuum generation, toroid microcavity, chalcogenide glasses, non-linear effects.

\section{INTRODUCTION}

Microresonators are structures that confine light in a micron scale area, mostly via the total internal reflection mechanism which results enhancing the electromagnetic energy at specific wavelengths determined by the interference effects present inside the medium. Among many different types of such cavities, microtoroids have attracted much attention since their first demonstration in 2003 [1], due to the possessed features of ultra high quality factor, small mode volume and integrability to chip based applications. Until now, such structures have been employed for variety of purposes including non-linear optical effects [2, 3, 4], laser action [5] and optical sensors [6].

Most of the time however, such toroidal microcavities with ultra high quality factor were only limited to $\mathrm{SiO}_{2}$ material since its fabrication is rather simple. This constraint therefore, led to working with other materials which are more advantageous in many aspects become scarce. In this paper we report an example of such a study that $\mathrm{SiO}_{2}$ material was merely used as a template for a chalcogenide material with a non-linear refractive index $\sim 1000$ times higher than that of silica, for observing the generation of new frequency components.

\section{FABRICATION}

First, microdisk patterns were transferred to thermally grown $\mathrm{SiO}_{2}$ layer on a silicon substrate using conventional lithography. Next using $\mathrm{SF}_{6}$ plasma the silicon layer underneath the microdisks was selectively and isotropically etched $\sim 10-25 \mu \mathrm{m}$. The undercut distance here is important for determining the major and minor diameter of the final toroid microcavity, Fig.1.

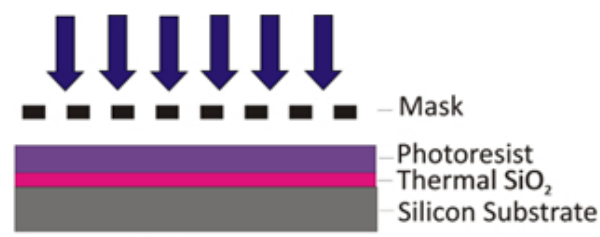

(a)

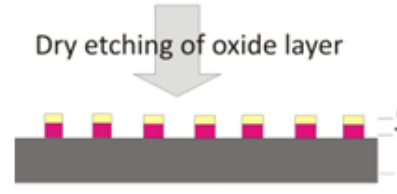

(c)

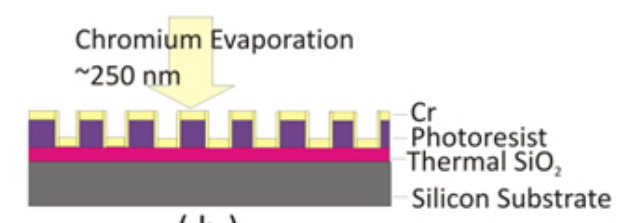

(b)

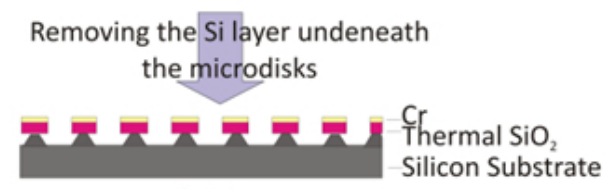

(d)

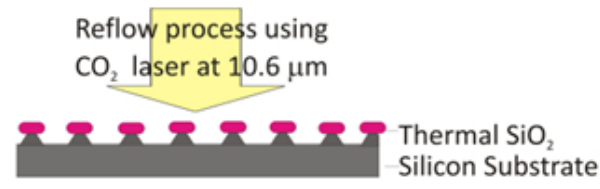

( e )

Figure 1. Fabrication steps for obtaining a silica microtoroid. 
Once microdisks with enough undercut were obtained, they were exposed to $\mathrm{CO}_{2}$ laser at $10.6 \mu \mathrm{m}$ for melting and letting them take the form of a smooth toroidal shape due to surface tension. In this process since the exposed area has a diameter of $\sim 200 \mu \mathrm{m}$, the silicon pillars were also exposed to the laser light, however they stay considerably cooler and therefore unaffected, because the silicon heat conductivity is much better than that of silica, Fig. 2.

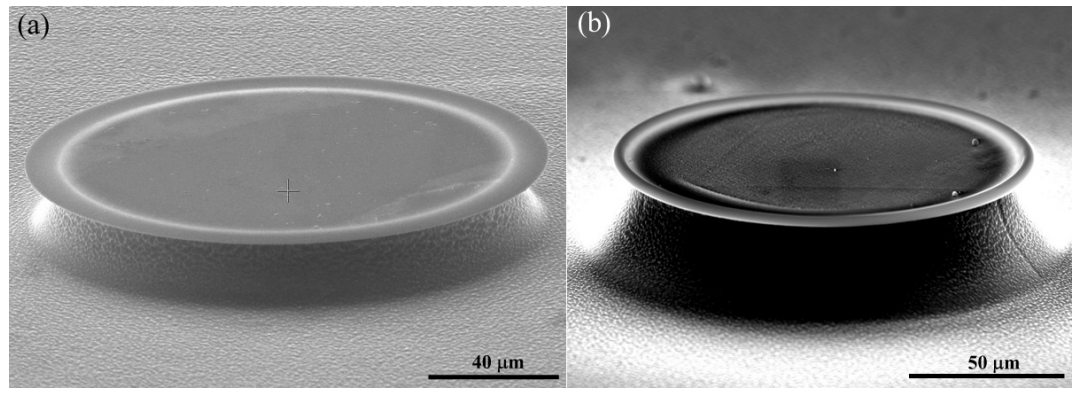

Figure 2. SEM images of the silica microdisk (a) before and (b) after the reflow process.

The surface roughness of the microcavities' rim is greatly reduced after the reflow process which is also verified by AFM measurements. From the results of such measurements, the surface roughness of the central region where no reflow effect occurred was found to be $\sim 2.4 \mathrm{~nm}$ on average; while the surface roughness on the rim of the toroid was less than $0.2 \mathrm{~nm}$, Fig. 3.

(a)

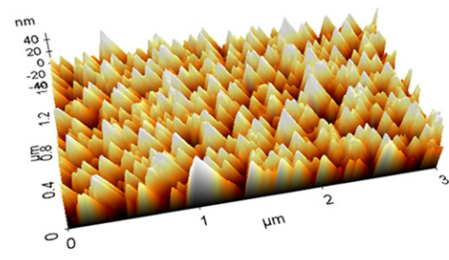

(b)

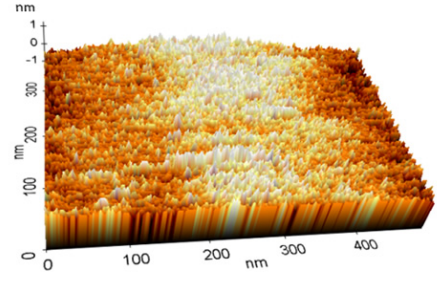

Figure 3. Surface profile of a microtoroid cavity (a) at the center and (b) at the rim.

An interesting phenomenon occurs when the pillar of the microdisk is so slim that it cannot hold the whole structure if it is exposed to laser illumination. In this case, the molten silica cannot be supported by the silicon pillar and part of it overflows down to substrate and the remaining takes the form of a microsphere, Fig. 4.
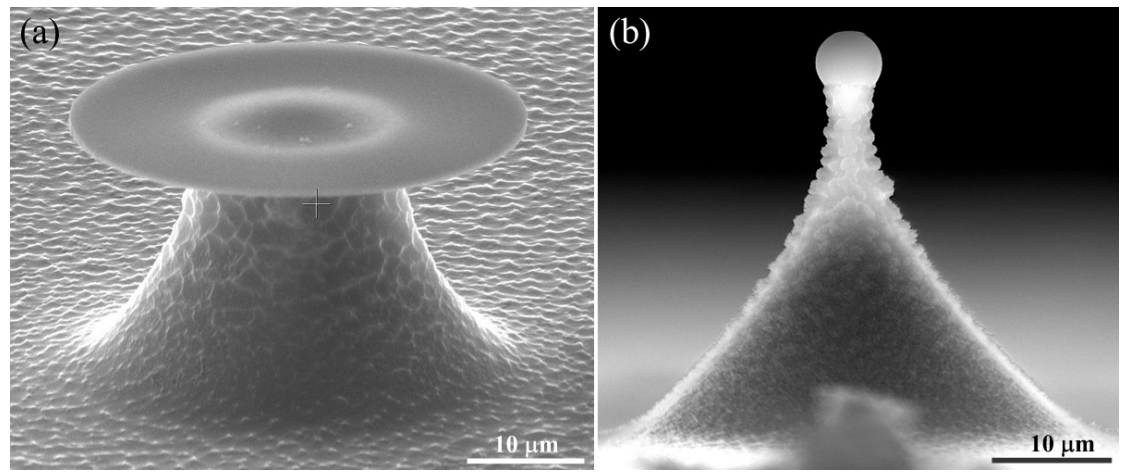

Figure 4. SEM images of the silica microdisk with $40 \mu \mathrm{m}$ diameter supported by $\sim 12 \mu \mathrm{m}$ diameter silicon pillar (a) before and (b) after the reflow process;

laser reflow results in a spherical shape. 
$\mathrm{As}_{2} \mathrm{~S}_{3}$, a chalcogenide material with very high nonlinear refractive index, was evaporated onto the obtained microtoroid structures with various thicknesses between 1 and $2 \mu \mathrm{m}$. The effect of evaporation to the surface roughness of the microcavity was minor which can be realized from SEM images in Fig. 5.
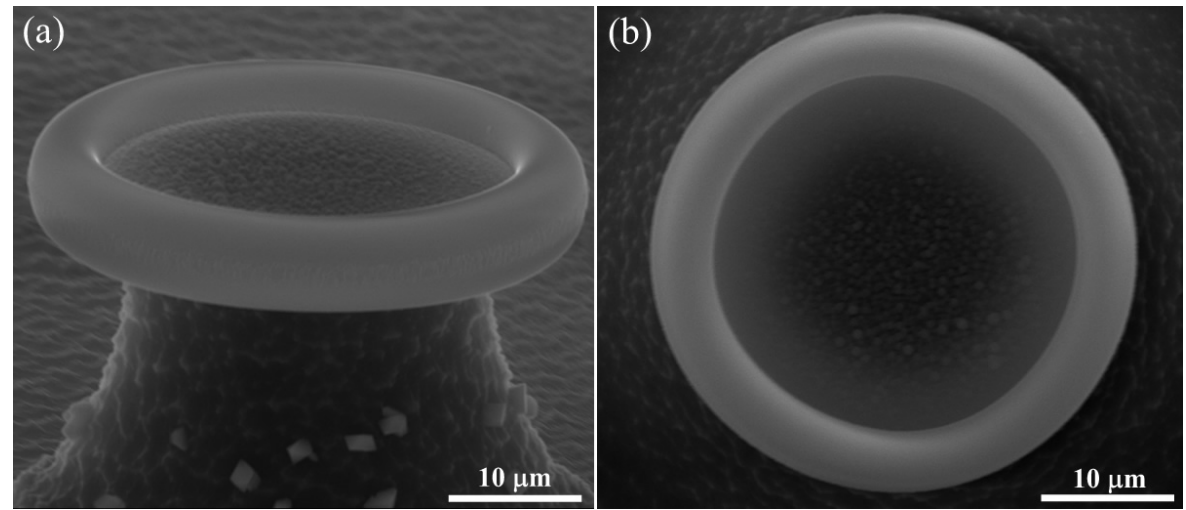

Figure 5. SEM images of the chalcogenide coated silica microtoroid (a) tilted and (b) from top.

\section{FDTD SIMULATIONS}

FDTD simulation results of the chalcogenide microtoroid structures have shown that new frequency components have been produced in the cavity due to non-linear processes of self phase modulation [7]. For this purpose, a microtoroid cavity having $30 \mu \mathrm{m}$ major diameter and $2 \mu \mathrm{m}$ minor diameter covered with $1.5 \mu \mathrm{m}$ thick chalcogenide layer of $\mathrm{As}_{2} \mathrm{~S}_{3}$ was designed. Cavity was excited at $1520 \mathrm{~nm}$ with approximately $100 \mathrm{fs}$ pulses. Modes are mostly confined in the non-linear material layer due to high refractive index contrast existing at the chalcogenide-air and chalcogenide-silica interfaces. Only 2-D simulations were performed due to limited power

(a)

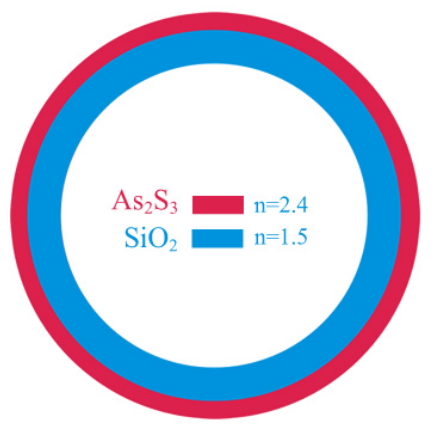

(b)

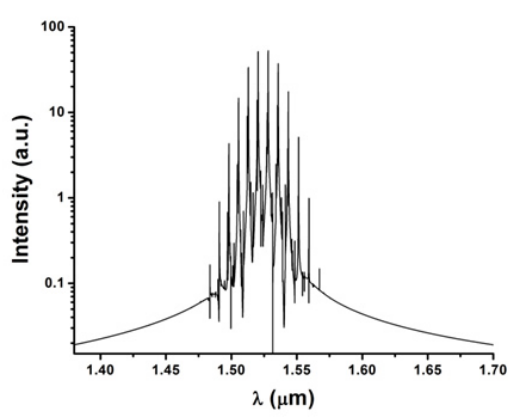

(c)

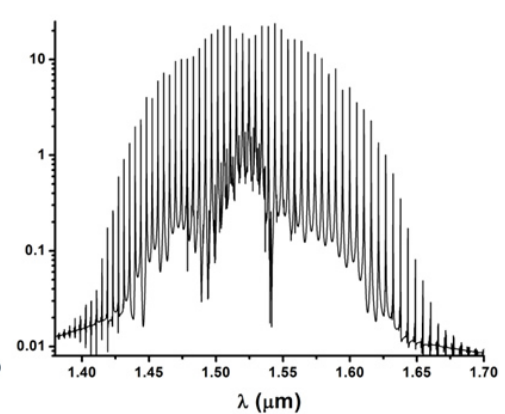

Figure 6. (a) Schematic illustration of the simulated structure where the blue regions are $\mathrm{SiO}_{2}$ and red regions are $\mathrm{As}_{2} \mathrm{~S}_{3}$. Simulation results for a (a) linear and (b) non-linear microtoroid.

of computation. Nevertheless it was enough to observe the non-linear effect of generating new frequencies around the excitation wavelength in a window of $\sim 300 \mathrm{~nm}$, Fig. 6 . Since the range of this window is also power dependent, obtaining a wider range is in principle possible once the excitation intensity is increased.

\section{CONCLUSIONS}

In summary we have fabricated chalcogenide microtoroid cavities maintaining ultra high quality factor by using $\mathrm{SiO}_{2}$ as a template, via conventional lithography techniques in conjunction with a reflow and evaporation procedure. Microcavities having $0.2 \mathrm{~nm}$ surface roughness could be obtained with this method. Simulation results have shown that, such a structure will produce new frequencies inside the microcavity in a wide range of spectrum due to non-linear interaction of the modes with the chalcogenide material.

\section{ACKNOWLEDGEMENTS}

This work was supported through Tubitak 106T348 and Turkish Academy of Sciences TUBA GEBIP awards. 


\section{REFERENCES}

[1] D.K. Armani, T.J. Kippenberg, S.M. Spillane, K.J. Vahala: Ultra-high-Q toroid microcavity on a chip, Nature, vol. 421. pp. 925-928, 2003.

[2] P. Del'Haye, A. Schliesser, O. Arcizet, T. Wilken, R. Holzwarth, T. J. Kippenberg: Optical frequency comb generation from a monolithic microresonator, Nature, vol. 450. pp. 1214-1217, 2007.

[3] A.A. Savchenkov, A.B. Matsko, L. Maleki: White light whispering gallery mode resonators, Optics Letters, vol. 31. pp. 92-94, 2006.

[4] M. Bayindir, A. F. Abouraddy, O. Shapira, et al:: Kilometer-long ordered nanophotonic devices by preform-to-fiber fabrication, IEEE J. Quan. Elec.,vol. 12. pp. 1202-1213, December 2006.

[5] L. Yang, T. Carmon, B. Min, et al.: Erbium-doped and Raman microlasers on a silicon chip fabricated by the sol-gel process, Appl. Phys. Lett., vol. 86. pp. 091114-3, February 2005.

[6] A. M. Armani and K. J. Vahala: Heavy water detection using ultra-high-Q microcavities, Opt. Lett., vol. 31. pp. 1896-1898, June 2006.

[7] G. P. Agrawal: Non-linear Fiber Optics, in Optics and Photonics, P. L. Kelley, Third Ed., New York: Academic Press, 2001. 